\section{Patterns of vitamin D, parathyroid hormone and c-terminal telopeptide of collagen type 1 in Caucasian and African descent HIV-infected populations in Central Europe}

\author{
Sebastian Noe, ${ }^{1}$ Celia Oldenbuettel, ${ }^{1}$ \\ Silke Heldwein, ${ }^{1}$ Hans Jaeger, ${ }^{1}$ \\ Eva Wolf ${ }^{2}$ \\ ${ }^{1}$ MVZ Karlsplatz, HIV Research and \\ Clinical Care Center, Munich; \\ ${ }^{2}$ MUC Research, Munich, Germany
}

\begin{abstract}
Risk factors for bone loss in HIV patients might differ or have a different impact in African descent compared to Caucasian populations. The aim of the paper is to analyze the relevance of risk factors on surrogate markers of bone metabolism in HIV-infected African descent and Caucasian patients. This is a cross-sectional study in a single HIV-specialized research and clinical care center in Munich, Germany. We included 889 patients in the study, among them 771 Caucasians (86.7\%). Only in Caucasians lower vitamin D levels [OR: 2.5 (95CI: 1.6-3.7)], lower calcium levels [OR: $1.8(1.2-2.8)$ ], and the use of tenofovir disoproxil fumarate [OR: 2.8 (1.8-4.4)] were significantly associated with elevated PTH in multivariate analysis. Likewise, only in Caucasians elevated PTH was significantly associated with elevated markers of c-terminal telopeptides of collagen type 1 ( $\beta$-CTX) [OR: $1.7(1.0-3.0)]$. Effects of traditional risk factors for secondary hyperparathyroidism and increased markers of bone turn-over seem to be less distinct in African descent HIV patients. The clinical impact and generalizability of this finding as well as the significance of vitamin D supplementation in African descent patients therefore warrants further investigation.
\end{abstract}

\section{Introduction}

HIV infected patients are known to be at a substantially higher risk of low bone mineral density (BMD) and fracture compared to non-infected controls with several traditional as well as HIV-specific risk factors seemingly contributing to this phenomenon. ${ }^{1,2}$ While it has been demonstrated that one of the most important risk factors - vitamin D insufficiency - seems to have a different impact on bone health in African compared to Caucasian subjects, ${ }^{3,4}$ the impact of other potential risk factors in African descent compared to Caucasian populations, particularly HIV-infected population, is even less clear and has not been studied in detail. A better understanding is yet of importance: the increasing migration from countries that include HIV highprevalence countries to areas like Central Europe or Northern America has confronted local HIV-caretakers with the uncertain relevance of findings like low vitamin $\mathrm{D}$, low calcium levels, and secondary hyperparathyroidism (sHPT) against the background of conflicting results of even basic therapeutic approaches like vitamin D supplementation in this subset of patients. ${ }^{3}$ Therefore, a better understanding seems to be important in order to identify patients who could potentially profit from any kind of intervention. This study aimed at describing potential risk factors of low BMD in Caucasian compared to African decent HIVinfected patients in Central Europe and at analyzing their association with surrogate markers of bone catabolism separately for both ethnicities.

\section{Materials and Methods}

This study was performed in a crosssectional design in a single HIV specialized outpatient center in Munich, Germany. Data was collected from clinical routine in the medical records of HIV patients, attending the clinic between January $1^{\text {st }}$ and December 31 $31^{\text {st }}$, 2016. All patients with proven HIV-infection and complete data for at least PTH, creatinine, vitamin D, AP, $\beta$ CTX, calcium, and phosphate and either Caucasian ethnicity or, for the African descent population, patients originating from African countries and living abroad in the first generation were selected. Patients with elevated creatinine levels $(\mathrm{N}=107)$, elevated calcium concentrations $(\mathrm{N}=0)$, unclear ART or within randomized clinical trials $(\mathrm{N}=32)$, on tenofovir alafenamide (TAF) containing ART $(\mathrm{N}=57)$, transgender patients $(\mathrm{N}=4)$, with non-Caucasian and non-African or unclear ethnicity $(\mathrm{N}=43)$, therapy with antiresorptive agents for osteoporosis $(\mathrm{N}=0)$, calcimimetics $(\mathrm{N}=0)$, as well as patients under vitamin $\mathrm{D}$ substitution $(\mathrm{N}=276)$ were excluded from the study. Demographic data, including date of birth, sex, current antiretroviral therapy, and laboratory parameters (creatinine, parathyroid hormone (PTH), vitamin D (25$\mathrm{OH}$-vitamin $\mathrm{D}_{3}$ ), $\beta$-CTX, AP, serum creatinine, albumin, serum calcium, and serum
Correspondence: Sebastian Noe, MVZ Karlsplatz, Karlsplatz 8, 80335 Munich, Germany.

Tel.: +49.89.5587030

E-mail: sno@jajaprax.de

Key words: HIV, vitamin D, PTH, African, $\beta$ crosslaps.

Acknowledgements: the authors would thank Dr. Matthew Page for carefully revising the manuscript.

Contributions: SN and EW developed the study concept and performed data analysis. All authors contributed in sampling and interpretation of data as well as the development and thorough revision of the manuscript

Conflict of interest: Dr. Noe and Dr. Heldwein report personal fees and non-financial support from Gilead, personal fees from ViiV, personal fees from AbbVie outside the submitted work. Dr. Oldenbuettel, Dr. Jaeger and Dr. Wolf report personal fees and non-financial support from Gilead, personal fees from ViiV, AbbVie, Bristol-Meyer Squibb, Janssen, and MSD Sharp and Dohme outside the submitted work.

Received for publication: 15 June 2017.

Revision received: 4 August 2017.

Accepted for publication: 7 August 2017.

This work is licensed under a Creative Commons Attribution-NonCommercial 4.0 International License (CC BY-NC 4.0).

(C) Copyright S. Noe et al., 2017

Licensee PAGEPress, Italy

Infectious Disease Reports 2017; 9:7265

doi:10.4081/idr.2017.7265

phosphate) were collected. For this study design with anonymous data from clinical routine no ethics committee's approval was required.

Unless otherwise declared, data are reported as median and interquartile range (IQR). For our primary objective, groups were compared for levels of PTH, AP, and $\beta$-CTX using Mann-Whitney test for unpaired samples, while the frequency of elevated $\beta$-CTX levels between both groups was compared using cross-tables and Fisher's exact test. Other comparisons of groups for continuous data were performed using Mann-Whitney test, while frequencies were compared by cross-tables and Fisher's exact test. For correlations, Spearman's correlation was used. In order to build a binary logistic regression model on the relation of elevated $\beta$-CTX and elevated PTH with the independent variables [age, vitamin D, calcium, phosphate, and PTH (for $\beta$-CTX)], independent variables 
were transformed into binary variables according to clinically important cut-off values at $<10$ (African) or $<20$ (Caucasian) $\mathrm{ng} / \mathrm{mL}, 2.20 \mathrm{mmol} / \mathrm{L},<2.6 \mathrm{mg} / \mathrm{dL}$, and $>65$ $\mathrm{pg} / \mathrm{mL}$ for low vitamin $\mathrm{D}$, low calcium, low phosphorus, and elevated PTH, respectively. Multivariate regression models were built in a stepwise forward approach, considering all variables with a $\mathrm{P}<0.25$ in univariate analysis for inclusion. Statistical analysis was performed using STATA SE 13.1 software (Stata, College Station, TX, USA). A $\mathrm{P}<0.05$ was considered as statistically significant for single testing, while Bonferroni correction was used to adjust for multiple testing. The manuscript was written according to STROBE guidelines for reporting observational studies. ${ }^{5}$

\section{Results}

Altogether, 889 patients were included in the study, $771(86.7 \%)$ of whom were Caucasian. The characteristics of the study population can be found in Table 1 .

In Caucasian patients, PTH was significantly (with $\mathrm{P}<0.013$ considered significant as adjusted for multiple testing) correlated with vitamin $D(\rho=-0.21 ; P<0.001)$ and calcium $(\rho=-0.21 ; P<0.001)$, but not with age $(\mathrm{P}=0.025)$ or phosphate $(\mathrm{P}=0.339)$. In African descent patients, PTH was significantly correlated with calcium $(\rho=-0.25$; $\mathrm{P}=0.007)$, but not with age $(\mathrm{P}=0.870)$, vitamin $\mathrm{D}(\mathrm{P}=0.385)$, or phosphate $(\mathrm{P}=0.209)$.

In Caucasian patients, $\beta$-CTX were significantly (with $\mathrm{P}<0.008$ considered significant as adjusted for multiple testing) correlated with PTH $(\rho=0.19 ; \mathrm{P}<0.001)$, but not with creatinine $(\mathrm{P}=0.024)$, calcium $(\mathrm{P}=0.066)$, age $(\mathrm{P}=0.524)$, vitamin $\mathrm{D}$ $(\mathrm{P}=0.083)$, or phosphate $(\mathrm{P}=0.355)$. In African patients, $\beta$-CL were not correlated with any of the parameters creatinine $(\mathrm{P}=0.086)$, age $(\mathrm{P}=0.386)$, vitamin $\mathrm{D}$ $(\mathrm{P}=0.970), \quad$ calcium $\quad(\mathrm{P}=0.647), \quad \mathrm{PTH}$ $(\mathrm{P}=0.097)$, or phosphate $(\mathrm{P}=0.281)$.

Fitted polynomial plots were used for an exploratory analysis of relations between vitamin D and PTH (Figure 1), vitamin D and $\beta$-CTX (Figure 2), as well as PTH and $\beta$-CTX (Figure 3).

Results of uni- and multivariate logistic regression analysis can be seen in Tables 2 and 3 .

\section{Discussion}

African populations, particularly from sub-Sahara areas, are known to have low vitamin D levels while, paradoxically, at the same time being at lower risk for osteoporo- sis and fracture. ${ }^{3,4}$ Whereas low vitamin D surely is an important, yet only single factor in the multifactorial genesis of impaired bone health in HIV-infected patients, ${ }^{2,6,7}$ little data is available on the epidemiology of other potential risk factors of low BMD in HIV-infected African descent populations in areas of higher latitude such as Central Europe. It is also unclear whether other risk factors might also contribute differently to impaired bone health in Caucasian and African descent subjects.

In this cohort of 889 HIV infected patients, in which we excluded major potential confounders on the variables of interest (impaired kidney function, use of antiresorptive therapy, calcimimetics, and vitamin D supplementation) with either

Table 1. Baseline characteristics of the study population per ethnicity.

\begin{tabular}{lccc} 
& Caucasian (n=771) & African (n=118) & P \\
Age, years & $49.4(40.8-56.0)$ & $37.7(30.8-46.4)$ & $<0.001^{*}$ \\
Male, N (\%) & $677(87.8)$ & $44(37.3)$ & $<0.001^{*}$ \\
\hline TDF-containing ART, N (\%) & $427(55.4)$ & $83(70.3)$ & $0.002^{*}$ \\
Creatinine, mg/dL & $0.97(0.83-1.08)$ & $0.80(0.66-0.95)$ & $<0.001$ \\
\hline Vitamin D, ng/dL & $22.1(14.5-29.1)$ & $12.9(7.6-19.3)$ & $<0.001$ \\
Vitamin D <30 ng/mL, N (\%) & $593(76.9)$ & $114(96.6)$ & $<0.001^{*}$ \\
\hline Vitamin D <20 ng/mL, N (\%) & $341(44.2)$ & $89(75.4)$ & $<0.001^{*}$ \\
Vitamin D <10 ng/mL, N (\%) & $101(13.1)$ & $43(36.4)$ & $<0.001^{*}$ \\
\hline PTH levels, pg/mL & $38.0(25.8-54.6)$ & $52.8(33.7-71.3)$ & $<0.001^{*}$ \\
PTH >65.0 pg/mL, N (\%) & $115(14.9)$ & $43(36.4)$ & $<0.001^{*}$ \\
\hline$\beta$-crosslaps, ng/mL & $0.33(0.24-0.47)$ & $0.33(0.23-0.50)$ & 0.777 \\
Elevated $\beta$-crosslaps, N (\%) & $64(1.2)$ & $10(8.5)$ & 1.000 \\
\hline Alkaline phosphatase, U/L & $80(66-97)$ & $74(65-98)$ & 0.236 \\
Elevated alkaline phosphatase, N (\%) & $133(17.3)$ & $13(11.0)$ & 0.089 \\
\hline Calcium, mmol/L & $2.24(2.17-2.30)$ & $2.21(2.15-2.28)$ & 0.052 \\
Calcium <2.20 mmol/L, N (\%) & $258(33.5)$ & $50(42.4)$ & 0.062 \\
\hline Phosphate, mg/dL & $3.3(3.0-3.7)$ & $3.6(3.2-3.9)$ & $0.001^{*}$ \\
Phosphate <2.6 mg/dL, N (\%) & $44(5.7)$ & $2(1.7)$ & 0.074 \\
\hline
\end{tabular}

Characteristics and laboratory results of patients included in this study per ethnicity. Unless otherwise declared, median and interquartile ranges are shown. *Significant differences between both groups on an $\alpha=0.05$

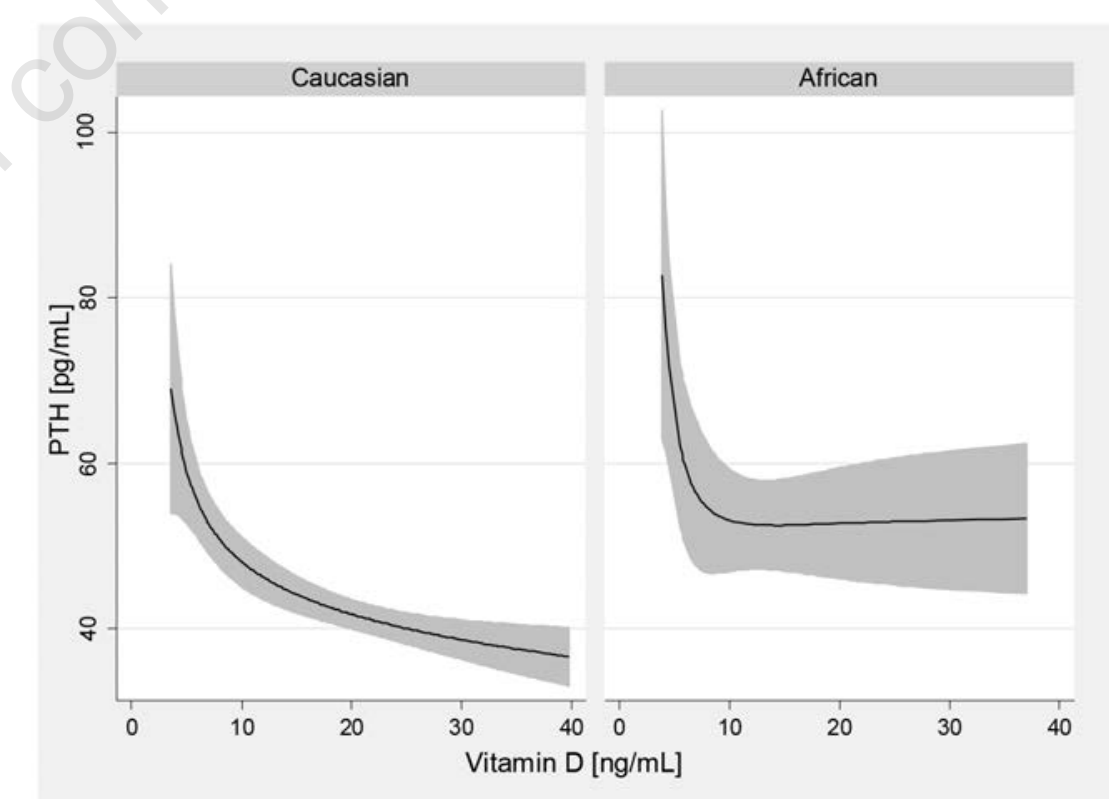

Figure 1. Fitted polynomial plots on the relation of vitamin D and PTH concentrations. The black line represents the fitted, polynomial smoothened association of vitamin D (25-OH-Cholecalciferol) with PTH concentration per ethnicity. The grey area represents the predicted $95 \%$ confidence interval. 
Caucasian or African ethnicity, we found that traditional risk factors of low bone mineral density - low vitamin $\mathrm{D}$, low calcium and, higher PTH levels with a higher frequency of (consecutive) secondary hyperparathyroidism - were more common in the African descent population, which is in good concordance with data from general African American populations in North America ${ }^{8-10}$ An exploratory, visual analysis also supported the assumption of different relations between vitamin $\mathrm{D}, \mathrm{PTH}$, and $\beta$ CTX (Figures 1-3) in the two ethnic groups. The most distinct difference seems to be found in the relation between vitamin D and PTH which will be discussed below.

The catabolic effect of PTH on bone is generally thought to be reflected by higher levels of markers of bone resorption, particularly telopeptides of collagen type 1 as described before. ${ }^{11-13}$ Yet, we could not verify suspected higher levels of $\beta$-CTX or a higher number of patients with elevated $\beta$ CTX in our African descent population when compared to the Caucasian.

In binary logistic regression models, we analyzed factors associated with elevated PTH levels and elevated $\beta$-CTX per ethnicity. While low vitamin D levels, low calcium levels, and use of tenofovir disoproxil fumarate (TDF) were associated significantly with elevated PTH levels in Caucasian patients, there were no significant associations in African patients. Similar findings were achieved in a multivariate analysis on elevated $\beta$-CTX, which found significant associations with PTH levels in the upper quartile together with use of TDF and gender for Caucasians, while only gender was a significant factor in the African population. These observations could imply that low vitamin D levels in African descent populations are of less effect on the development of secondary

hyperparathyroidism (sHPT) compared to Caucasian patients. An overall different association of vitamin D and PTH levels can be seen in Figure 1: whereas for the

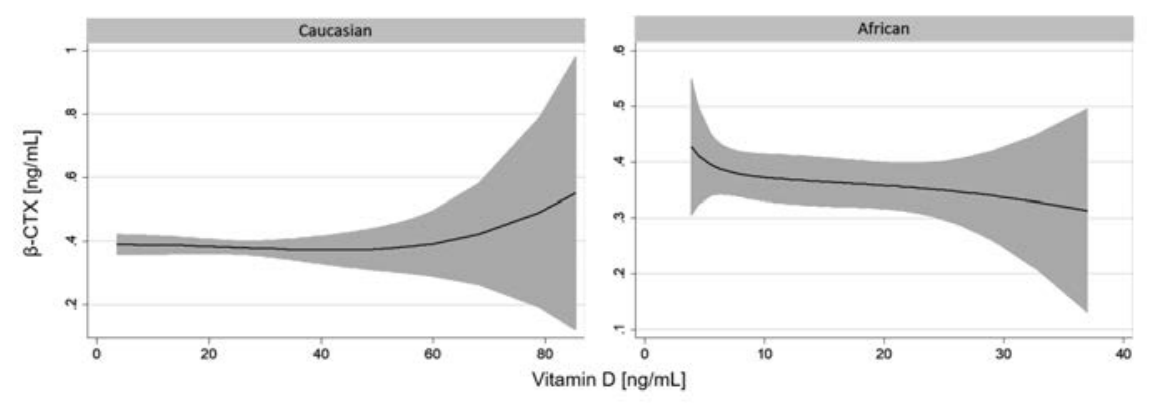

Figure 2. Fitted polynomial plots on the relation of vitamin D and $\beta-C T X$ concentrations. The black line represents the fitted, polynomial smoothened association of vitamin D (25-OH-Cholecalciferol) with $\beta$-CTX concentration per ethnicity. The grey area represents the predicted $95 \%$ confidence interval.

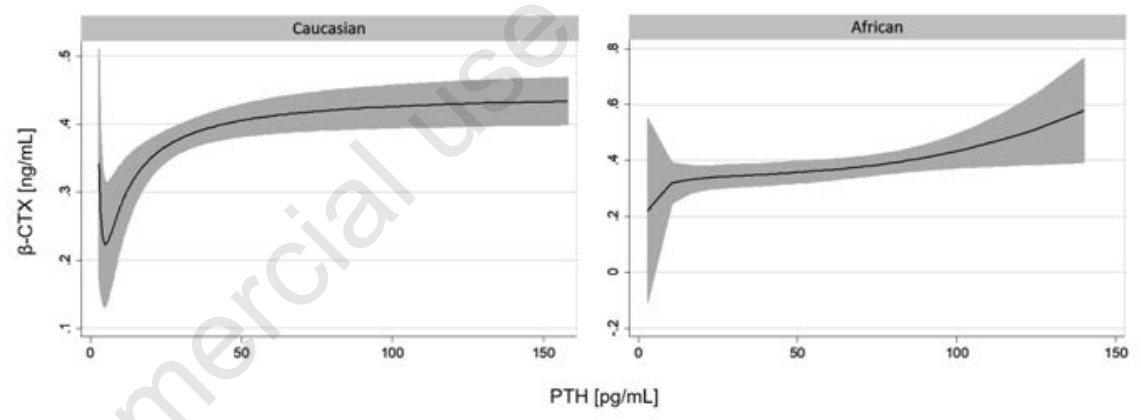

Figure 3. Fitted polynomial plots on the relation of PTH and $\beta-C T X$ concentrations. The black line represents the fitted, polynomial smoothened association of PTH with $\beta$-CTX concentration per ethnicity. The grey area represents the predicted $95 \%$ confidence interval.

Table 2. Association of different binary factors on the occurrence of elevated PTH in uni- and multivariate logistic regression analysis analyzed per ethnicity.

\begin{tabular}{|c|c|c|c|c|c|c|}
\hline & \multicolumn{3}{|c|}{ Univariate } & \multicolumn{3}{|c|}{ Multivariate } \\
\hline & OR & $95 \mathrm{CI}$ & $\mathbf{P}$ & OR & $95 \mathrm{CI}$ & $\mathbf{P}$ \\
\hline \multicolumn{7}{|c|}{ Caucasian patients } \\
\hline Female gender & 1.4 & $0.8-2.5$ & 0.284 & & & \\
\hline Age in the highest quartile & 0.8 & $0.5-1.2$ & 0.254 & & & \\
\hline Vitamin D levels in the lowest quartile & 2.3 & $1.5-3.5$ & $<0.001$ & 2.5 & $1.6-3.7$ & $<0.001$ \\
\hline Serum calcium concentration $<2.20 \mathrm{mmol} / \mathrm{L}$ & 1.9 & $1.3-2.8$ & 0.002 & 1.8 & $1.2-2.8$ & 0.004 \\
\hline TDF containing ART & 2.7 & $1.8-4.3$ & $<0.001$ & 2.8 & $1.8-4.4$ & $<0.001$ \\
\hline NRTI-free ART & 0.7 & $0.4-1.2$ & 0.179 & & & \\
\hline \multicolumn{7}{|c|}{ African decent patients } \\
\hline Female gender & 0.6 & $0.3-1.5$ & 0.329 & & & \\
\hline Age in the highest quartile & 1.6 & $0.7-3.6$ & 0.272 & & & \\
\hline Vitamin D levels in the lowest quartile & 2.1 & $0.9-4.8$ & 0.087 & & & \\
\hline Serum calcium concentration $<2.20 \mathrm{mmo} / \mathrm{L}$ & 1.3 & $0.6-2.8$ & 0.491 & & & \\
\hline TDF containing ART & 1.1 & $0.5-2.6$ & 0.752 & & & \\
\hline NRTI-free ART & 1.1 & $0.4-2.9$ & 0.862 & & & \\
\hline
\end{tabular}

Since characteristics of subgroups were different for age and vitamin D, the 4th quartile was determined for each population separately. 
Table 3. Association of different binary factors on the occurrence of elevated $\beta$-crosslaps in uni- and multivariate exact logistic regression analysis analyzed per ethnicity.

\begin{tabular}{|c|c|c|c|c|c|c|}
\hline & \multicolumn{3}{|c|}{ Univariate } & \multicolumn{3}{|c|}{ Multivariate } \\
\hline & OR & $95 \mathrm{CI}$ & $\mathbf{P}$ & OR & $95 \mathrm{CI}$ & $\mathbf{P}$ \\
\hline \multicolumn{7}{|c|}{ Caucasian patients } \\
\hline Female gender & 0.1 & $0.0-0.6$ & 0.004 & 0.1 & $0.0-0.7$ & 0.024 \\
\hline Age in the highest quartile & 0.5 & $0.3-1.0$ & 0.051 & & & \\
\hline Vitamin D levels in the lowest quartile & 1.3 & $0.7-2.3$ & 0.345 & & & \\
\hline Serum calcium concentration $<2.20 \mathrm{mmol} / \mathrm{L}$ & 1.1 & $0.6-1.8$ & 0.847 & & & \\
\hline PTH levels in the highest quintile & 1.8 & $1.1-3.1$ & 0.025 & 1.7 & $1.0-3.0$ & 0.046 \\
\hline Tenofovir containing ART & 1.8 & $1.1-3.2$ & 0.027 & 1.7 & $1.0-2.9$ & 0.069 \\
\hline NRTI-free ART & 0.6 & $0.3-1.3$ & 0.203 & & & \\
\hline \multicolumn{7}{|c|}{ African decent patients } \\
\hline Female gender & 0.03 & $0.0-0.2$ & $<0.001$ & 0.03 & $0.0-0.2$ & $<0.001$ \\
\hline Age in the highest quartile & 1.1 & $0.3-4.4$ & 0.931 & & & \\
\hline Vitamin D levels in the lowest quartile & 1.2 & $0.3-4.8$ & 0.831 & & & \\
\hline Serum calcium concentration $<2.20 \mathrm{mmol} / \mathrm{L}$ & 0.3 & $0.1-1.5$ & 0.153 & & & \\
\hline PTH levels in the highest quintile & 1.2 & $0.3-5.1$ & 0.780 & & & \\
\hline Tenofovir containing ART & 4.1 & $0.5-34.0$ & 0.186 & & & \\
\hline NRTI-free ART & 0.5 & $0.1-4.1$ & 0.509 & & & \\
\hline
\end{tabular}

Since characteristics of subgroups were different for age, vitamin D, and PTH, the 4th quartile was determined for each population separately

Caucasian population we found an association that is in line with observations as published before, ${ }^{14}$ the association in the African population is characterized by a plateau of PTH levels over a wide span of vitamin $\mathrm{D}$ levels, whereas only vitamin $\mathrm{D}$ levels $<10 \mathrm{ng} / \mathrm{mL}$ result in a strong increase in PTH.

This only seems to be one aspect, as even the development of SHPT in African descent patients might result in a lesser degree in bone catabolic activity as indicated by $\beta$-CTX when compared to Caucasians. This is in line with what has been reported before from general African descent populations in western countries. $3,15-17$

In the context of HIV our findings are of particular interest: evidence suggests that effects of TDF might be exerted by higher levels of PTH in patients on TDF-containing therapies. ${ }^{18-20}$ This could imply that TDF-associated and PTH-mediated effects on bone might also be less pronounced in (African and) African descent populations, whereas continuation of the use of TDF (not only against the background of recently published positive cardiovascular data $)^{21}$ might be desirable at least in a subset of patients for several reasons. Therefore, further investigation is warranted whether African patients profit from non-TDF [including tenofovir alafenamide (TAF)] regimens in the same way as recently demonstrated in general populations. ${ }^{22,23} \mathrm{Of}$ note, patients on TAF were excluded for analysis in our study, as due to the recent introduction of TAF into clinical routine we had to suspect a selection bias on those patients that had already been changed.

Our study has several limitations. First, the cross-sectional design is of disadvantage since we cannot state on unmeasured confounders like time of patients outside their home countries or time on ART, whereas some adverse effects might only develop after some time being exposed to lower sun irradiation as well as other life style or environmental factors. Still, both PTH and $\beta$-CTX have been shown to promptly react to changes in bone metabolism. ${ }^{24}$ Second, we cannot provide data on changes on BMD or occurrence of fracture. Evaluation of different degradation products of telopeptides of collagen type 1 has yet been shown to reflect bone catabolism in patients with high PTH levels and correlate with (particularly cortical) bone mass. ${ }^{25-27}$ Third, the subgroup of African descent patients is much smaller than the Caucasian subgroup. The wide range of some of the 95CIs indicate that a larger number of patients in this subgroup might be needed for a better estimation of the real effects in some of our secondary endpoints, particularly as far as the use of TDF is concerned. The African population might therefore not be powered to detect effects of similar size in the African compared to the Caucasian population. Another issue is the reliability of total vitamin D concentrations in African populations who were described to have lover levels of vitamin D binding protein (VDB) compared to Caucasians. ${ }^{28}$ Recent findings suggest that these observations might have been due to the use of monoclonal antibodies, possibly due to VDB polymorphisms in African population. However, the use of polyclonal antibody assays did not add any benefit compared to the assessment of total vitamin $\mathrm{D}$ and results were highly correlated $(\mathrm{r}=0.80$ 0.83). ${ }^{29,30}$ Fourth, to get a better impression of bone turn-over, more surrogate markers of bone turnover (including bone-specific alkaline phosphatase, P1NP) would have been desirable, but where not available from routine data in our cohort. We still think that due to our findings and the mechanism of action of PTH, a marker for bone resorption should be more important to assess the question of its impact on bone. ${ }^{11-13}$ A potential further issue concerns the daily and seasonal variability of many of the markers under investigation; although patients are generally advised to have blood samples taken fasting, there is no possibility to state on the fasting status of patients which might be of particular interest for $\beta$ CTX. We did also not adjust for the season of blood sampling due to suspected collinearity of vitamin D and season, whereas we adjusted for vitamin D on an analytic level in our regression models. Last, one might argue about the impact of the differences in baseline characteristics of both populations, that limit the epidemiologic statement. Yet, for major questions we 
used intra-ethnicity analysis and therefore adjusted on an analytic level.

These considerations are also limiting the generalizability of our data. Furthermore, as many environmental factors are of significant influence on bone metabolism (sunlight exposure, nutrition, physical activity and many more) it seems questionable if results can be extrapolated to populations in other regions of the world. Also, the exclusion of patients with elevated creatinine limits the generalizability for patients with (even mildly) impaired kidney function, although bone metabolism is of particular interest in this subset of patients. The exclusion of patients on TAF-based regimens furthermore questions the generalizability for patients under treatment with this new tenofovir prodrug.

\section{Conclusions}

Associations between vitamin D and PTH levels were found to be overall different in African patients. We found traditional risk factors of negative bone balance such as low calcium and vitamin D levels or high levels of PTH to be more prevalent but not to be significantly associated with markers of bone catabolism in African descent HIVpatients. Yet our results are limited by the relatively small sample size of the African population. In order to confirm our findings, studies in larger cohorts are strongly encouraged. Since data on the outcome of vitamin D supplementation in African patients is conflicting, ${ }^{3}$ more data is also needed on the right indications for vitamin D supplementation in this subset of patients.

Another interesting aspect of our observation is that higher levels of $\beta$-CTX were associated with the use of TDF only in Caucasian patients, which certainly need further investigation in bigger cohorts. Since our data derive from a single center in Central Europe, there are some restrictions concerning generalizability of the absolute values of our data since they might be strongly depending on environmental factors and latitude. It also remains unclear, whether or not our data can be generalized to non-HIV-populations.

\section{References}

1. Compston J. Osteoporosis and fracture risk associated with HIV infection and treatment. Endocrinol Metabol Clin North Am 2014;43:769-80.

2. Compston J. HIV infection and osteoporosis. Bonekey Rep 2015;4:636.

3. O'Connor MY, Thoreson CK, Ramsey NL, et al. The uncertain significance of low vitamin D levels in African descent populations: a review of the bone and cardiometabolic literature. Prog Cardiovasc Dis 2013;56:261-9.

4. Aloia JF. African Americans, 25hydroxyvitamin D, and osteoporosis: a paradox. Am J Clin Nutr 2008;88:545s$50 \mathrm{~s}$.

5. von Elm E, Altman DG, Egger M, et al. The Strengthening the Reporting of Observational Studies in Epidemiology (STROBE) statement: guidelines for reporting observational studies. Lancet 2007;370:1453-7.

6. Triant VA, Brown TT, Lee H, Grinspoon SK. Fracture prevalence among human immunodeficiency virus (HIV)-infected versus non-HIV-infected patients in a large U.S. healthcare system. J Clin Endocrinol Metabol 2008;93:3499-504.

7. McComsey GA, Huang JS, Woolley IJ, et al. Fragility fractures in HIV-infected patients: need for better understanding of diagnosis and management. J Int Assoc Physicians AIDS Care (Chic) 2004;3:86-91.

8. Yao S, Hong CC, Bandera EV, et al. Demographic, lifestyle, and genetic determinants of circulating concentrations of 25-hydroxyvitamin D and vitamin D-binding protein in African American and European American women. Am J Clin Nutr 2017;105: 1362-71.

9. Shieh A, Aloia JF. Assessing bitamin D status in African Americans and the influence of vitamin D on skeletal health parameters. Endocrinol Metab Clin North Am 2017;46:135-52.

10. Schleicher RL, Sternberg MR, Lacher $\mathrm{DA}$, et al. The vitamin D status of the US population from 1988 to 2010 using standardized serum concentrations of 25-hydroxyvitamin D shows recent modest increases. Am J Clin Nutr 2016;104:454-61.

11. Takami H, Ikeda Y, Hayashi K, et al. Clinical assessment of collagen crosslinked N-telopeptides as a marker of bone metabolism in patients with primary hyperparathyroidism. Biomed Pharmacother 1999;53:329-33.

12. Harris SS, Soteriades E, DawsonHughes B. Secondary hyperparathyroidism and bone turnover in elderly blacks and whites. J Clin Endocrinol Metabol 2001;86:3801-4.

13. De la Piedra C, Diaz Martin MA, Diaz Diego EM, et al. Serum concentrations of carboxyterminal cross-linked telopeptide of type I collagen (ICTP), serum tartrate resistant acid phosphatase, and serum levels of intact parathyroid hormone in parathyroid hyperfunction. Scand J Clin Lab Invest 1994;54:11-5.

14. Holick MF, Siris ES, Binkley N, et al. Prevalence of Vitamin D inadequacy among postmenopausal North American women receiving osteoporosis therapy. J Clin Endocrinol Metabol 2005;90:3215-24.

15. Gutierrez OM, Farwell WR, Kermah D, Taylor EN. Racial differences in the relationship between vitamin $\mathrm{D}$, bone mineral density, and parathyroid hormone in the National Health and Nutrition Examination Survey. Osteoporos Int 2011;22:1745-53.

16. Cosman F, Shen V, Morgan D, et al. Biochemical responses of bone metabolism to 1,25-dihydroxyvitamin D administration in black and white women. Osteoporos Int 2000;11:271-7.

17. Cosman F, Morgan DC, Nieves JW, et al. Resistance to bone resorbing effects of PTH in black women. J Bone Miner Res 1997;12:958-66.

18. Masia M, Padilla S, Robledano C, et al. Early changes in parathyroid hormone concentrations in HIV-infected patients initiating antiretroviral therapy with tenofovir. AIDS Res Hum Retroviruses 2012;28:242-6.

19. Patricio JA Jr., Lopes PF, Medeiros T, et al. Tenofovir monotherapy for hepatitis $\mathrm{B}$ after 1 year does not produce renal dysfunction, but is associated with hyperparathyroidism not related to vitamin D. Eur J Gastroenterol Hepatol 2016;28:64-9.

20. Rosenvinge MM, Gedela K, Copas AJ, et al. Tenofovir-linked hyperparathyroidism is independently associated with the presence of vitamin D deficiency. J Acquir Immune Defic Syndr 2010;54:496-9.

21. Chen R, Scherzer R, Hsue PY, et al. Association of tenofovir use with risk of incident heart failure in $\mathrm{HIV}$-infected patients. J Am Heart Assoc 2017;6.

22. Sax PE, Zolopa A, Brar I, et al. Tenofovir alafenamide vs. tenofovir disoproxil fumarate in single tablet regimens for initial HIV-1 therapy: a randomized phase 2 study. J Acquir Immune Defic Syndr 2014;67:52-8.

23. Pozniak A, Arribas JR, Gathe J, et al. Switching to Tenofovir Alafenamide, coformulated with elvitegravir, cobicistat, and emtricitabine, in HIV-infected patients with renal impairment: 48week results from a single-arm, multicenter, open-label phase 3 study. J Acquir Immune Defic Syndr 2016;71:530-7.

24. Chubb SA. Measurement of C-terminal 
telopeptide of type I collagen (CTX) in serum. Clin Biochem 2012;45:928-35.

25. Woitge HW, Pecherstorfer M, Li Y, et al. Novel serum markers of bone resorption: clinical assessment and comparison with established urinary indices. $\mathrm{J}$ Bone Miner Res 1999;14:792-801.

26. Cortet B, Cortet C, Blanckaert F, et al. Bone ultrasonometry and turnover markers in primary hyperparathyroidism. Calcif Tissue Int 2000;66:11-5.
27. Christiansen P, Steiniche T, Brixen K, et al. Primary hyperparathyroidism: whole-body bone mineral density in surgically treated Danish patients: a three-year follow-up study. Bone 1999;25:597-602.

28. Powe CE, Evans MK, Wenger J, et al. Vitamin D-binding protein and vitamin D status of black Americans and white Americans. N Engl J Med 2013;369:1991-2000.

29. Nielson CM, Jones KS, Chun RF, et al.
Free 25-hydroxyvitamin D: Impact of vitamin $\mathrm{D}$ binding protein assays on racial-genotypic associations. J Clin Endocrinol Metabol 2016;101:2226-34. 30. Jemielita TO, Leonard MB, Baker J, et al. Association of 25-hydroxyvitamin D with areal and volumetric measures of bone mineral density and parathyroid hormone: impact of vitamin D-binding protein and its assays. Osteoporos Int 2016;27:617-26. 